\title{
Exposición ocupacional a plomo y cadmio en personal de salud
}

JULIA GONZÁLEZ(1), GONZALO ALFARO(2), MARÍA PAZ ARRIAGADA(2), CARMEN CASTILLO(3), VERÓNICA MUÑOZ(4), JUAN PABLO ESPINA(4) y WALDO ARANDA(1)

\section{RESUMEN}

Con el fin de contribuir a evitar las consecuencias potencialmente graves derivadas de la exposición crónica a plomo y cadmio, se describe la situación de exposición ocupacional a estos metales de un grupo de trabajadores de un hospital público de la Región Metropolitana. Se describen parámetros bioquímicos relacionados con la exposición a $\mathrm{Pb}$ y $\mathrm{Cd}$ al momento de detectarse el problema y al cabo de dos años de haber implementado medidas protectoras. Se trata de un estudio de cohorte histórico único, usando información secundaria. Inicialmente se encuentra niveles promedio de plomo en sangre, ZPP y cadmio en orina dentro del rango normal; después de la intervención se observa una variación significativa en estos parámetros lo cual señala la efectividad de la intervención preventiva. Los exámenes funcionales de órganos o sistemas potencialmente afectados por la exposición no muestran alteraciones ni variaciones significativas pre y post intervención. Se pone en evidencia que las intoxicaciones por plomo y cadmio son un problema presente en el ámbito laboral chileno y que las medidas de protección aplicadas a los trabajadores son eficaces, especialmente si se detecta precozmente el problema, antes de que se evidencien repercusiones derivadas de la intoxicación crónica.

Palabras Claves: Plomo, cadmio, exposición ocupacional, Intoxicación.

\section{ABSTRACT \\ OCCUPATIONAL EXPOSURE TO LEAD AND CADMIUM AMONG HEALTHCARE WORKERS}

In order to contribute to the prevention of the potentially serious consequences of chronic exposure to lead $(\mathrm{Pb})$ and cadmium $(\mathrm{Cd})$, the current status of occupational exposure to these metals among a group of workers of a Public Hospital of the Metropolitan Area of Santiago is described.

Biochemical parameters related to the exposure to $\mathrm{Pb}$ and $\mathrm{Cd}$ were measured at the time of problem detection and two years after the implementation of preventive measures. The present is a study involving a unique historic cohort, using secondary information. Initial findings were average blood levels of lead and ZPP and cadmium in urine within the normal range; after the preventive intervention a significant variation in these parameters was observed,

(1) Escuela de Salud Pública, Facultad de Medicina, Universidad de Chile.juliagonzalez@med.uchile.cl

(2) Escuela de Medicina. Facultad de Medicina. Universidad de Chile

(3) Unidad de Medicina del Trabajo. CDT Dra. Eloísa Díaz. Complejo Hospitalario Norte.

(4) Departamento de Salud Ocupacional. Secretaria Regional Ministerial. Región Metropolitana. 
thus indicating the effectiveness of the intervention. Functional testings of the organs potentially affected by the exposure did not show alterations or significant variations between pre and postpreventive intervention analysis. The present work demonstrates that that lead and cadmium intoxications are a current problem within the Chilean healthcare occupational environment and that preventive measures implemented to protect workers are effective, especially if the problem is detected early, before chronic intoxication-derived consequences are observed.

Key Words: Lead, Cadmium, occupational exposure, intoxication.

\section{INTRODUCCIÓN}

A principios del 2002, se confeccionaban en un hospital de la Región Metropolitana protecciones para pacientes con tratamientos de radioterapia, usando un material de aleación fusible denominado Cerrobend, que contenía plomo, estaño, cadmio y bismuto, metales que pueden afectar la salud de quienes trabajan con ellos.

La confección de dichas protecciones se efectuaba en una sala denominada "Bodega de Cerrobend", $\left(5 \mathrm{~m}^{2}\right.$ y $3 \mathrm{~m}$ de alto), sin ventilación natural ni artificial. La fundición del Cerrobend se realizaba en una olla, calentada por una cocinilla, y posteriormente la fusión era depositada sobre moldes. Luego se limaban las protecciones para liberar asperezas.

Las instalaciones fueron visitadas por el organismo regulador de la época -Unidad de Salud Ocupacional del Servicio de Salud correspondiente-, constatándose que las personas que trabajaban en dicha bodega ignoraban los efectos tóxicos de estos metales, no utilizaban guantes ni protección respiratoria y los delantales usados al momento de hacer los moldes eran los mismos que usaban en sus otras actividades y de propiedad del funcionario, por lo que su limpieza dependía de este último.

Finalmente, y según protocolos establecidos, el organismo fiscalizador efectuó los exámenes de rigor a los trabajadores expuestos y realizó una intervención consistente en:

- Usar ropa protectora: gorro, guantes, protección respiratoria y antiparras. - Usar casilleros exclusivos para almacenar ropa de trabajo.

- Limpiar ropa de trabajo aisladamente.

- Recolectar diariamente la escoria producto del proceso de fusión.

- Minimizar derrames y salpicaduras, que producen polvos y residuos.

- Limitar el proceso de limado, pues produce exceso de polvo.

- Siempre utilizar aspirador para limpieza del lugar.

- No comer, beber ni fumar en la sala de moldeo.

- Asear prolijamente piel y cabello mediante una ducha y cambiarse ropa después de terminado el trabajo.

- Verificar diariamente que los elementos de extracción y ventilación funcionen adecuadamente.

- Capacitar al menos una vez al año al personal encargado de fabricar los moldes de aleación fusible respecto de medidas de protección.

Al cabo de 2 años de iniciada la intervención, se controló a los trabajadores realizándose los exámenes de laboratorio pertinentes.

El plomo no tiene función biológica útil en el hombre y en su forma natural tiene poca importancia como fuente de contaminación. Ésta se alcanza en el ámbito ocupacional y ambiental, considerándose la intoxicación por plomo como una de las enfermedades de origen profesional y ambiental más graves, por su alta prevalencia, gran penetración ambiental y toxicidad persistente en los afectados. Puede llegar a la mayoría de los órganos y en niños es importante el daño del sistema nervioso central ${ }^{1}$. Al organismo puede ingresar por vía digestiva, respiratoria o dérmica.

Las fuentes de contaminación ocupacional por plomo son: la minería; la fundición y la industria. La contaminación ambiental por plomo se asocia a la combustión de gasolina y la fundición primaria de plomo ${ }^{2}$.

El cadmio es un metal no esencial para el hombre, presente en los tejidos por exposición ambiental o laboral. Puede ingresar al organismo 
por vía respiratoria, digestiva o cutánea.

La exposición laboral se observa en: la industria; refinamiento de zinc; fundición; producción y derivados de cadmio; fórmulas de color seco para plásticos, etc. Entre las exposiciones no ocupacionales están las ambientales: contaminación de agua, aire, suelo, productos alimenticios y humo de cigarrillo.

En Chile las publicaciones que documentan intoxicaciones laborales por plomo hacen referencia a casos clínicos individuales ${ }^{3}$ o casos de contaminación ambiental producida por fábricas que trabajan con dicho elemento, demostrando que tanto los trabajadores como las personas que habitan en las cercanías de la fábrica presentan niveles más altos que el resto de la población ${ }^{4}$. La Asociación Chilena de Seguridad (ACHS) describió las intoxicaciones diagnosticadas y atendidas en sus establecimientos entre los años 1990 - 1999, encontrándose que la intoxicación laboral por plomo ocupó el $6^{\circ}$ lugar dentro de los agentes más frecuentes ${ }^{5}$. También se han documentado niveles elevados de plomo en poblaciones infantiles de Antofagasta y lactantes de la Región Metropolitana, cuyo origen es ambiental ${ }^{6-8}$, así como un caso de saturnismo en una niña expuesta a plomo en un taller de baterías 9 .

No se han descrito en Chile casos de intoxicación exclusiva por cadmio ni por ambos metales; sin embargo, existen en la literatura internacional casos de intoxicación por plomo y cadmio en trabajadores de oficinas de reparación de baterías y en pescadores expuestos a polución de una fundición de plomo, ambos en Brasil ${ }^{10,11}$.

La hipótesis del trabajo es que la exposición laboral a plomo y cadmio puede ocasionar aumento de los niveles plasmáticos y urinarios de estos metales, y eventualmente alterar órganos o sistemas. Y a su vez, esto puede ser revertido a través de la instauración de medidas que eviten el impacto de esta exposición en la salud del trabajador.

El propósito del trabajo es contribuir a sensibilizar a posibles expuestos y sus empleadores, para evitar las consecuencias potencialmente graves desarrolladas por la exposición crónica a plomo y cadmio.

\section{OBJETIVOS}

El objetivo general del estudio es describir parámetros bioquímicos relacionados con exposición a $\mathrm{Pb}$ y $\mathrm{Cd}$ en trabajadores laboralmente expuestos y evaluar el impacto de las medidas de protección implementadas para prevenir o revertir los efectos en salud de dicha exposición.

Los objetivos específicos son:

- Describir perfil sociodemográfico de los trabajadores expuestos.

- Describir niveles de exámenes para la detección de plomo y cadmio en sangre y orina.

- Describir resultados de exámenes funcionales de órganos o sistemas potencialmente afectados por dicha exposición.

- Evaluar el impacto de la intervención preventiva en los niveles de plomo y cadmio en sangre y orina y en hemograma y perfil hepático.

\section{MATERIAL Y MÉTODO}

Se realizó un estudio de cohorte histórico único, usando información secundaria. Se cuenta con una medición inicial de los parámetros bioquímicos y funcionales, realizada al momento de tomar conocimiento de la situación.

Posteriormente, se realizó una intervención en los trabajadores y en el lugar de trabajo, con el objetivo de reducir la exposición a plomo y cadmio. Después de dos años se repitieron los exámenes.

Las fuentes de información utilizadas fueron las fichas clínicas y los protocolos creados por el organismo fiscalizador, que evalúan posibles exposiciones previas a plomo y cadmio, sintomatología de exposición y medición de parámetros de laboratorio.

La población estudiada corresponde al universo de trabajadores asignado por la institución para desempeñar funciones en una bodega destinada a la fundición de Cerrobend, de un Hospital de la R.M, durante el año 2002. Ésta corresponde a 12 trabajadores: 6 técnicos paramédicos y 6 tecnólogos médicos. Se 
excluyeron del estudio aquellos que no trabajaban directamente en la fundición de Cerrobend.

Los exámenes fueron realizados en tres laboratorios: hematocrito, hemoglobina, bilirrubina total, fosfatasas alcalinas, GOT y GPT fueron efectuados por el Laboratorio A el año 2002 y por el Laboratorio B el año 2004; en tanto que $\mathrm{Pb}$ en sangre, zincprotoporfirina (ZPP) y $\mathrm{Cd}$ en orina se efectuaron en el Laboratorio $\mathrm{C}$ en ambas oportunidades. Los métodos utilizados fueron:

\section{Laboratorio A:}

- Hemograma: máquina Sysmex KX21N, Roche, Sysmex Corporation.

- Pruebas Hepáticas: máquina Automatic Analyzer 912, Roche, Hitachi, Japón.

\section{Laboratorio B:}

- Hemograma: máquina Colter STKS, año 1990, Deadline Miami, USA

-Pruebas hepáticas: máquina Modular PE, Roche, Hitachi, Japón.

\section{Laboratorio C:}

- Plomo en sangre: A.A.G.HidrurodA.A.H.Grafito ColorimetríaHematofluorómetro.

Los valores normales utilizados para el análisis de los datos fueron los entregados por el laboratorio B para hemograma y pruebas hepáticas; y por el laboratorio $\mathrm{C}$ para cadmio en orina, ZPP y plomo en sangre.
Para el análisis de datos se calcularon los promedios de los parámetros de laboratorio y se compararon los valores obtenidos antes y después de la intervención y sus respectivos valores normales. A continuación se seleccionaron los exámenes que mostraron mayores variaciones (Cadmio en Orina, Plumbemia y ZPP) y se compararon los resultados individuales pre y post intervención. Con el objeto de identificar variaciones significativas, dado el pequeño tamaño de muestra, se usó una técnica no paramétrica de comparación para muestras pareadas, el test de Wilcoxon, con un nivel de significación de $0,05^{12}$.

\section{RESULTADOS}

De los 12 trabajadores expuestos a plomo y cadmio en el año 2002, se analizaron los datos de 9 de ellos; excluyéndose 3 por no presentarse a la reevaluación ni realizarse los exámenes de control el año 2004. Su edad varía entre 27 y 43 años con promedio 34.4; 4 de los 9 son mujeres. Hay 4 Técnicos Paramédicos y 5 Tecnólogos Médicos.

Los promedios de todos los exámenes se encuentran en el rango de normalidad. Sin embargo, tres pacientes presentan valores anormales en ambas mediciones (antes y después de la intervención): B presenta bilirrubina total alterada; I presenta Fosfatasas alcalinas alteradas; y G presenta GPT alterada (ver promedios y rangos en tabla 1).

Tabla 1. Resultados de Exámenes de Laboratorio años 2002 y 2004

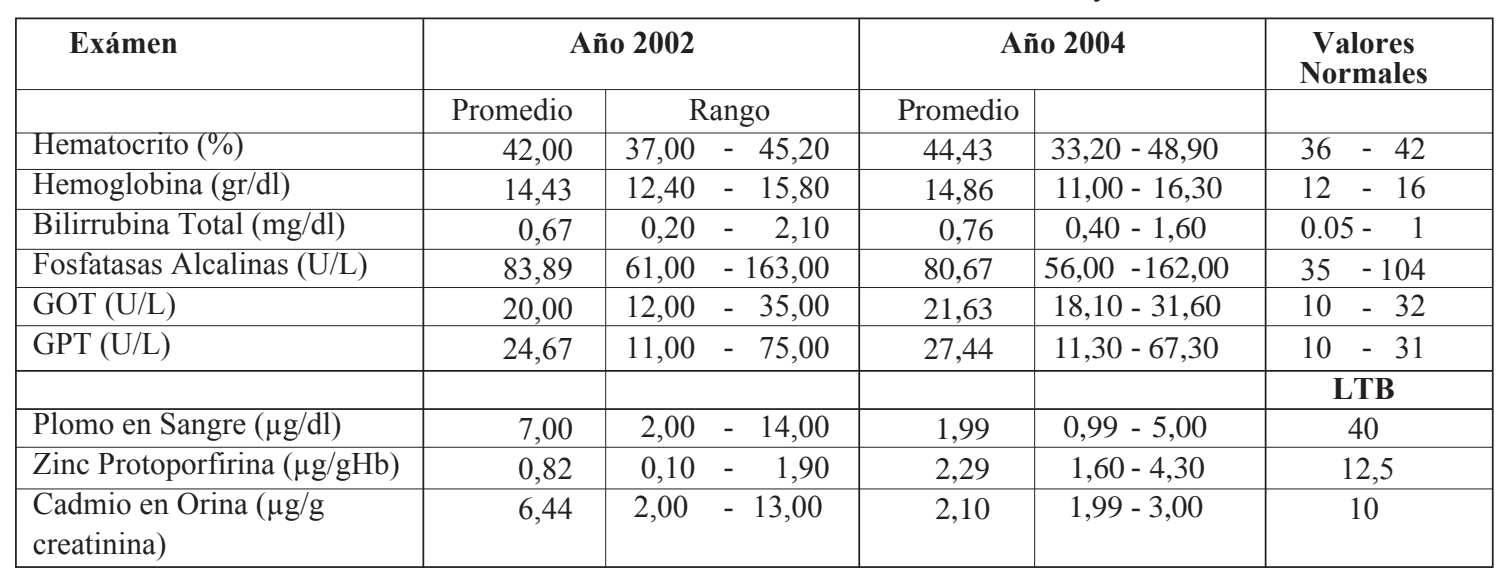


El promedio de los resultados de las pruebas que pesquisan alteraciones funcionales, no muestra diferencias entre los años 2002 y 2004. En cambio, los niveles promedio de plomo en sangre, ZPP y cadmio en orina muestran una variación importante entre antes y después de la intervención; por este motivo se analizan las variaciones individuales pre y post-intervención; precisamente los exámenes realizados en un mismo laboratorio, antes y después de la intervención.

\section{Plomo en Sangre}

Al comparar los valores individuales de plomo en sangre pre y post-intervención se encuentran diferencias significativas $(\mathrm{p}<0.0151)$; ocho de los nueve trabajadores disminuyeron la concentración de plomo en sangre. (Tabla 2).

Tabla 2. Niveles individuales de Plomo en Sangre año 2002 y 2004

\begin{tabular}{|c|r|r|}
\hline PACIENTE & $\begin{array}{r}\text { PLOMO AÑ 2002 } \\
\text { (LTB: 40 ug/d) }\end{array}$ & $\begin{array}{r}\text { PLOMO AÑ 2004 } \\
\text { (LTB: 40 ug/dl) }\end{array}$ \\
\hline $\mathrm{A}$ & 3,00 & 5,00 \\
\hline $\mathrm{B}$ & 7,00 & 0,99 \\
\hline $\mathrm{C}$ & 11,00 & 2,00 \\
\hline $\mathrm{D}$ & 4,00 & 0,99 \\
\hline $\mathrm{E}$ & 6,00 & 3,00 \\
\hline $\mathrm{F}$ & 9,00 & 0,99 \\
\hline $\mathrm{G}$ & 14,00 & 3,00 \\
\hline $\mathrm{H}$ & 7,00 & 0,99 \\
\hline $\mathrm{I}$ & 2,00 & 0,99 \\
\hline Promedio & 7,00 & 1,99 \\
\hline
\end{tabular}

\section{Zinc Proto Porfirina (ZPP)}

$\mathrm{Al}$ analizar las variaciones individuales de ZPP pre y postintervención se encuentran diferencias significativas $(\mathrm{p}<0.0077)$. Todos los trabajadores aumentaron el nivel sanguíneo de ZPP. (Tabla 3).

\section{Cadmio en Orina}

Al analizar las variaciones individuales de Cadmio en orina pre y postintervención se encuentran diferencias significativas $(p<0.0076)$. Todos los trabajadores disminuyeron el nivel (Tabla 4).
Tabla 3. Niveles individuales de Zinc Protoporfirina años 2002 y 2004

\begin{tabular}{|c|r|r|}
\hline PACIENTE & $\begin{array}{r}\text { ZPP año 2002 } \\
\text { (LTB: 12,5 } \\
\text { ug/grHb) }\end{array}$ & $\begin{array}{r}\text { ZPPaño 2004 } \\
\text { (LTB: 12,5 } \\
\text { ug/grHb) }\end{array}$ \\
\hline A & 0,60 & 2,00 \\
\hline B & 1,90 & 2,10 \\
\hline C & 0,10 & 2,20 \\
\hline D & 0,10 & 1,60 \\
\hline E & 1,00 & 4,30 \\
\hline F & 0,50 & 1,80 \\
\hline G & 0,80 & 1,90 \\
\hline H & 1,90 & 2,60 \\
\hline I & 0,50 & 2,10 \\
\hline Promedio & 0,82 & 2,29 \\
\hline
\end{tabular}

Tabla 4. Niveles individuales de Cadmio en Orina años 2002 y 2004

\begin{tabular}{|c|r|r|}
\hline PACIENTE & $\begin{array}{r}\text { CADMIO EN } \\
\text { ORINA AÑO 2002 } \\
\text { (LTB:10ug/gr Creatinina) }\end{array}$ & $\begin{array}{r}\text { CADMIO EN } \\
\text { ORINA AÑO 2004 } \\
\text { (LTB:10ug/gr Creatinina) }\end{array}$ \\
\hline $\mathrm{A}$ & 9,00 & 1,99 \\
\hline $\mathrm{B}$ & 5,00 & 1,99 \\
\hline $\mathrm{C}$ & 13,00 & 1,99 \\
\hline $\mathrm{D}$ & 4,00 & 1,99 \\
\hline $\mathrm{E}$ & 4,00 & 1,99 \\
\hline $\mathrm{F}$ & 2,00 & 1,99 \\
\hline $\mathrm{G}$ & 8,00 & 1,99 \\
\hline $\mathrm{H}$ & 11,00 & 3,00 \\
\hline $\mathrm{I}$ & 2,00 & 1,99 \\
\hline Promedio & 6,44 & 2,10 \\
\hline
\end{tabular}

\section{DISCUSIÓN}

Según el Decreto Supremo 109 sobre calificación y evaluación de accidentes del trabajo y enfermedades profesionales, plomo y cadmio se consideran agentes de riesgo en la legislación chilena. Por este motivo, el trabajo realizado en la bodega de Cerrobend podría constituir un factor de riesgo para el desarrollo de una enfermedad profesional en los trabajadores expuestos ${ }^{13}$.

A pesar de que la población afectada tiene un nivel educacional alto, los trabajadores no tenían conocimiento de los metales a los que estaban expuestos, ni del riesgo que implicaba dicha exposición. Situación, además, grave por tratarse de una población joven y con una exposición potencialmente prolongada. 
Entre las consecuencias de la intoxicación por plomo destacan: síntomas generales, como decaimiento, fatiga, tos, impotencia sexual, temblor; alteraciones de los sistemas hematopoyético, nervioso central y periférico, urinario, gastrointestinal, cardiovascular, reproductor, endocrino; alteraciones en crecimiento y desarrollo intrauterino.

La intoxicación crónica por cadmio produce alteraciones en los sistemas respiratorio, nefrourológico y musculoesquelético; así como cáncer pulmonar, próstatico, renal y gástrico; y altera la fertilidad y presión arterial.

Al consultar dirigidamente, los pacientes no refirieron síntomas de intoxicación por plomo o cadmio. Los exámenes que evaluaban función de órganos no mostraron alteraciones en ninguna de las dos mediciones, lo que significa que no se alcanzó a dañar la función al menos de sistema hematopoyético e hígado. Para evaluar la exposición a cadmio se pueden utilizar: cadmio en orina, sangre, heces y/o raíz del pelo. Se usó la primera, ya que es un buen reflejo de la carga corporal del metal, y al aproximarse a un nivel crítico, refleja una exposición aguda ${ }^{14}$.

De los indicadores de exposición a plomo (plumbemia, plumburia y plumburia provocada) se utilizó la plumbemia, por ser un buen reflejo del grado de exposición ${ }^{15}$. La plumbemia alcanza rápidamente una meseta, aún cuando la cantidad de plomo en el organismo continúe aumentando, y aunque el trabajador sea alejado de la fuente de contaminación, este metal puede seguir ejerciendo su acción tóxica.

La Zinc Protoporfirina eritrocitaria es un indicador que evalúa el efecto del plomo; comienza a aumentar en exposiciones constantes una vez que el plomo ha alcanzado niveles de $35-40 \mu \mathrm{g} / 100 \mathrm{ml}$. Se utilizó por su sensibilidad, especificidad, bajo costo y facilidades en la toma de muestra.

En Chile, el Decreto Supremo No 594/00 del Ministerio de Salud, actualmente vigente, que aprueba el reglamento sobre condiciones sanitarias y ambientales básicas en los lugares de trabajo, dispone que el valor límite para plomo en sangre es $40 \mu \mathrm{g} / 100 \mathrm{ml}$, para cadmio en orina es $10 \mu \mathrm{g} / \mathrm{g}$ creatinina, y para ZPP es $12.5 \mu \mathrm{g} / \mathrm{gHb}^{16}$.

No obstante, según la Ocupational Safety \&
Health Administration (OSHA), organismo encargado de proponer normas internacionales en salud ocupacional, los trabajadores con un nivel de cadmio en orina menor a $3 \mu \mathrm{g} / \mathrm{g}$ creatinina ya están en riesgo y deben ser evaluados al menos anualmente por un médico.

Los trabajadores con niveles entre 3 y $7 \mu \mathrm{g} / \mathrm{g}$ creatinina y con niveles mayores, deben controlarse con parámetros bioquímicos y evaluación médica, mejorar las condiciones de trabajo, removerse del puesto de trabajo y excluirse obligatoriamente de sus actividades. Esto se justifica ya que niveles mayores a $5 \mu \mathrm{g} / \mathrm{g}$ creatinina, implican saturación de todos los otros tejidos con cadmio ${ }^{14,17}$.

En el caso del plomo, la OSHA señala que el trabajador con más de $40 \mu \mathrm{g} / 100 \mathrm{ml}$, ya se encuentra en riesgo y debe ser notificado por su empleador en forma escrita. Además recomienda la remoción del puesto de trabajo en forma temporal o permanente, según el grado de intoxicación ${ }^{18,19}$.

El año 2002, el promedio de plomo en sangre fue de $7 \mu \mathrm{g} / 100 \mathrm{ml}$, el de ZPP de $0,82 \mu \mathrm{g} / \mathrm{gHb}$ y el de cadmio en orina de $6.44 \mu \mathrm{g} / \mathrm{g}$ creatinina, valores promedio dentro de la norma, pero con algunos trabajadores que sobrepasaban incluso los límites legales chilenos (pacientes $\mathrm{C} \mathrm{y} \mathrm{H}$ ).

Es destacable el descenso en los niveles individuales de plomo y cadmio entre la primera y segunda medición, en todos los casos, lo que sugiere que las medidas propuestas son eficaces para evitar el ingreso de estos metales al organismo y prevenir una posible repercusión orgánica, constituyendo una propuesta que debería ser difundida en los centros de trabajo con riesgos de exposición.

El aumento de ZPP observado en la segunda medición puede explicarse porque existiría un período de latencia para que los cambios en el nivel de plomo se expresen en este parámetro; un cambio en las tareas o grado de exposición se reflejarán inmediatamente en la plumbemia, pero el cambio en la ZPP tardará en producirse ${ }^{20}$.

Esto último refleja que los trabajadores estudiados, muestran efectos latentes de la exposición a plomo aún cuando se hayan aplicado medidas eficaces para disminuir su ingreso al organismo. 


\section{CONCLUSIONES}

Las intoxicaciones por plomo y cadmio son un problema presente en el ámbito laboral chileno, pero no existen registros que muestren su real magnitud y contribuyan a entregar una visión global del problema.

Aún así, hay documentados varios casos de intoxicación ambiental por plomo y cadmio y en menor proporción, reportes de intoxicación laboral a nivel nacional e internacional.

Se ha demostrado en el presente trabajo, que las medidas de protección aplicadas a los trabajadores son eficaces para disminuir el ingreso de estos metales al organismo en poblaciones expuestas laboralmente a plomo y cadmio. Sobretodo sí, como en esta situación, se detecta precozmente el problema, antes de que se evidencien repercusiones derivadas de la intoxicación crónica, situación éticamente más aceptable que esperar que los trabajadores sobrepasen los límites para empezar a actuar frente a esta exposición grave y evitable es necesario crear conciencia del problema y sus soluciones tanto en empleadores y trabajadores, como en equipos de salud.

\section{REFERENCIAS}

1. HOWSON P, HERNÁNDEZ M, RALL P. Introducción. En: El plomo en América: Estrategias para la prevención. México: Instituto Nacional de Salud Pública (INSP), Instituto Nacional de Medicina de Estados Unidos, 1996; 17-21.

2. COREY G, GALVAO L. Plomo, Serie Vigilancia, 8. Metepec, Edo. de México: Centro Panamericano de Ecología Humana y Salud, OPS/OMS. 1989; 5-63.

3. DE LA TORRE JM. Intoxicación plúmbica: Saturnismo. Bol. Hosp. San Juan de Dios. 1997; 44(3): $167-171$.

4. QUIJADA, I., ESTRADA, P., TAGER, N. Estudio de contaminación ambiental por plomo en la comuna de Freire. Rev. Med. Sur. 1986; 11(3): 118-121.

5. MORENO G, ROMEO J. Intoxicaciones diagnosticadas en ACHS 1990 - 1999. Bol. Cient. Asoc. Chil. Segur. 2000;2(3): 55 - 58.

6. SEPÚlVEDA V, VEGA J, DELGADO I. Exposición severa a plomo ambiental en una población infantil de Antofagasta, Chile. Rev. Med. Chile. 2000;128(2): 221-32.

7. FRENZ P, VEGA J, MARCHETTI N, TORRES J, KOPPLIN E, DELGADO I, et al. Exposición crónica a plomo ambiental en lactantes chilenos. Rev. Med. Chile. 1997;125(10): 1137-1144.

8. VEGA J, FRENZ P, MARCHETTI N, TORRES J, KOPPLIN E, DELGADO I, et al. Exposición crónica a plomo ambiental en lactantes chilenos II: Efectos en el desarrollo psicomotor. Rev. Med. Chile. 1999; 127(1): $28-37$.

9. LOPETEGUI F, LLORENTE J. Intoxicación Plúmbica (saturnismo). Pediatr. Día. 1987; 3(4): 218-221.

10. CARVALHO F, SILVANY-NETO A, CHAVES M, MASCARENHAS T, ALT F. Intoxicaçao por chumbo e cadmio en trabalhadores de oficinas para reforma de baterias em salvador. Rev. saúde pública. 1985;19(5):411-20.

11. CARVALHO F, MASCARENHAS T, SOUZA S, LINHARES P. Absorçao e intoxicaçao por chumbo e cadmio em pescadores da regiao do Rio Subae. Ciênc. Cult. (Säo Paulo). 1983;

35(3): 360-366.

12. WILCOXON F. Individual Comparisons by Ranking Methods. En: Biometrics 1. 1945. 80-83.

13. Decreto Supremo No 109 de 7 de junio de 1968 del Ministerio del Trabajo. Decreto Supremo No 594 de 15 de septiembre de 1999 del Ministerio de Salud.

14. RAMÍREZ A. Toxicología del Cadmio. Conceptos Actuales para evaluar exposición ambiental u ocupacional con indicadores biológicos. Anales de la Facultad de Medicina, Universidad Nacional Mayor de San Marcos. 2002;63(1): 51-64.

15. ALESSIO L, FOA V. Human biological monitoring of industrial chemicals series.En: Lead. Ed: Comission of the European Communities; 1983.

16. ASOCIACIÓN CHILENA DE SEGURIDAD ACHS. Normas Legales sobre Accidentes de Trabajo y Enfermedades Profesionales. 2002.

17. OCCUPATIONAL SAFETY \& HEALTH ADMINISTRATION. Toxic and Hazardous Substances, Substance Safety Data Sheet - Cadmium. 1993.

18. OCCUPATIONAL SAFETY \& HEALTH ADMINISTRATION. Toxic and Hazardous Substances Lead. 2005.

19. ARRATE M., RODRÍGUEZ-SIERRA N, MARTÍNEZ A. Protocolos de vigilancia sanitaria específica: Plomo. Madrid: Edita Ministerio de sanidad y consumo, 1999; 1-52.

20. VERSSCHOOR M, HERBER R, ZIELHUIS R, WIBOWO A. Zinc protoporphyrin as an indicator of lead exposure: precision of zinc protoporphyrin measurements. Int Arch Occup Environ Health. 1987; 59: 613-621.

Ud. puede comentar éste y otros artículos publicados en la Revista de Salud Pública, enviando un correo electrónico a revistasp@med.uchile.cl 